\title{
Modeling of the Time Structure of Construction Processes Using Neural Networks
}

DOI 10.1515/otmcj-2016-0018

Received September 15, 2016; accepted June 17, 2017

\begin{abstract}
The aim of this research is to develop a methodology for calculating the standard time using neural networks. The paper presents research questions and proposes a methodology. In addition, the methods used to calculate the standard time in the Czech Republic and in other parts of the world are presented, and the basics of the mathematics of neural networks are shortly explained. The paper also provides an example that represents a simple application of a neural network. This example is designed to confirm some assumptions and to arrive at the basic conclusions regarding the operation of models created using neural networks.
\end{abstract}

Keywords: construction technology, time scheduling, neural networks

\section{Introduction}

\subsection{Research motivation}

If we look to the future of project information modeling, building information modeling (BIM) is certainly the next standard for the design and maintenance of buildings. Therefore, there is still much progress to be made. The accuracy and applicability of the model directly depend on the amount of detail and quality of information that is written directly into the properties of the individual functional elements of the model. Basic element information includes element geometry, physical characteristics, data

\footnotetext{
*Corresponding author: Čeněk Jarský, Department of Construction Technology, Faculty of Civil Engineering, Czech Technical University in Prague, Praha, Czech Republic, E-mail: jarsky@contec.com Kateřina Petlíková, Department of Construction Technology, Faculty of Civil Engineering, Czech Technical University in Prague, Praha, Czech Republic, E-mail: petlikova.katerina@gmail.com
}

that determines the appearance and behavior of the elements. Some information is easy to determine, whereas for others, we need to have enough time for calculations and experimental measurements. One of these properties is the standard time.

Standard time is the amount of time that a laborer or machine consumes to meet one unit of measurement in a given process. It is therefore an important indicator in the preparation of construction schedules, building budgets, and the monitoring of resource needs. The aim of the authors' research is to develop a methodology for determining the standard time using neural networks (NNs).

\subsection{Overview of current methods}

Current methods used to determine the standard time (statistical, multiple regression analysis [MRA] and casebased reasoning [CBR]) have many shortcomings, which are described for each method individually. NNs appear to be an appropriate method to remove some of the shortcomings of the methods currently in use.

In recent years, several studies have been dedicated to the possibilities of using NNs for estimating the construction cost (Kim et al. 2004; Arditi and Tokdemir 1999; Attalla and Hegazy 2003; Petroutsatou et al. 2012). Some of these studies include not only the results of the application of NNs but also comparisons with the previously mentioned methods (MRA, CBR). NNs are proving to be more convenient and accurate. The problem is that there is the need to train NNs and determine their architecture first. This process is very tedious. The studies deal with the buildings as a whole and do not focus on the specific construction processes. Moreover, the studies are focused on the construction cost. The chosen parameters are simple, e.g., floor area, number of floors, type of facility and quality standards. The studies do not go into detail on other aspects.

Other research is devoted to the use of NNs in the construction industry, e.g., in the field of risk analysis (Xu et al. 2017), localization of errors in the created model (Berkhahn and Tilleke 2008) or static calculations (Goh 1998). 


\subsection{The aims of the paper}

The aims of the paper are as follows:

1. To present research questions and propose a methodology for future research

2. To provide an overview of methods currently used to determine the standard time

3. To present current applications of NNs in construction engineering

4. To present methods used to calculate the standard time in the Czech Republic and in the world

5. To present the basic mathematics of NNs

6. To provide an example that represents application of NN

\section{Research questions and proposed methodology}

Research is at an early stage, the purpose of the example in this paper is to take a closer look into the structure and performance of NNs, as well as to confirm certain assumptions (mentioned in sections 1.2, 5.1 and 6.0). The next step is to work in the field, where it is necessary to measure and record sufficient time data found during the implementation of the selected process. Once the data is collected, it will be divided into two groups. The first set of data will be used to calculate the standard time by all currently used methods as well as by the authors' proposed method using $\mathrm{NN}$. The second data group will serve to test the error rates of these methods. The methods will be compared from selected aspects (time requirement, accuracy of result, computational difficulty, and so on). Finally, a multicriteria comparison of the methods will be performed.

Given the need for very close support from practice, it is not yet certain what kind of data and how much data can be obtained. The objective is to obtain time data of several similar simple processes in the field of steel structures, which would serve to verify the basic assumptions, the initial development of the basic methodology and the introduction of basic questions. Detailed analyses and comprehensive solutions to the issue would be the subject of further research.

For the development and testing, steel structures were selected. In addition to the atypical structure, with sufficient experience, they are predictable. Steel structures often consist of repetitive elements and operations. There is absence of wet process. Moreover, experts from the practice are interested in collaboration on research.

\section{Work standardization methods currently used}

\subsection{Methods used in the Czech Republic}

Methods for determining standard times in the Czech Republic are used when creating databases of standard times for budgeting software. Both methods are statistical. Thus, a sufficient number of processes are measured and recorded, e.g., we take time frames, and then the average standard time is statistically evaluated. They can be divided into two basic groups according to the scale of analysis of the monitored process:

- Analytical methods

- Summary methods

Analytical methods determine the standard times in steps. First, it is necessary to divide the working process into individual working operations. The next step is to determine the standard time of these operations and the work breaks and, from this data, to calculate the standard time of the whole process by the simple summing up of the average values of all working operations.

Summary methods determine the standard times as a whole, without prior analysis of the monitored process. For example, we measure a hundred times the amount of time that the bricklayer spends with $150 \mathrm{~mm}$ thick porous walls, and we use the average value in the database.

The shortcomings of these methods are connected with the fact that they are not dealing with the dependency of the output (standard time) on the parameters of the specific project (e.g., extent and complexity of work, weather, worker experience, and so on). Databases contain person-hours calculated from the standard times, which are not possible to edit, according to conditions that occur for a particular project. Even if we had wanted to make the revision ourselves, on the basis of the observed dependencies, we would lack information about the conditions under which the database was measured, so the starting point is not given to us.

\subsection{Methods used in other parts of the world}

In the Czech Republic, standard times are exclusively part of a commercial database, and only a few construction companies manage their own database. In other regions of the world, it is more common that companies manage their own databases and do not rely as much on a third party. One of the reasons is that higher wages are 
offered abroad, which affect the cost more. Therefore, more-accurate methods were developed, which took into account the input parameters. The most widely used are MRA and CBR.

\subsubsection{MRA model}

MRA (Eq.1) is used in situations in which we are interested in the dependency of certain quantitative (continuous) variables on one or more other quantitative (continuous) variables called regressors. The first step is to establish the independent and dependent variables. The goal of regression analysis is to describe this dependence using an appropriate model. For example, we can write down the duration for each working process (dependent variable) and the conditions that were met during the measurement (independent variables: e.g., extent and complexity of work, weather, worker experience, and so on). After we find out the mathematical dependence of the variables, we can calculate the new values according to the following formula:

$$
\mathrm{Y}=\mathrm{C}+\mathrm{b}_{1} \mathrm{X}_{1}+\mathrm{b}_{2} \mathrm{X}_{2}+\cdots+\mathrm{b}_{\mathrm{n}} \mathrm{X}_{\mathrm{n}}
$$

Where $\mathrm{Y}$ is predictable from regressors $\mathrm{X}_{1}, \mathrm{X}_{2}, \ldots \mathrm{X}_{\mathrm{n}}$. C is a constant, and $b_{1}, b_{2}, \ldots b_{n}$ are coefficients (Kim et al. 2004).

\subsubsection{CBR model}

The essence of CBR (Eq. 2) lies in the fact that the researcher approaches the problem by trying to find its similarity with all the problems solved and successfully resolved before. The target is not to seek a new and unique solution for a new problem but to deduce it by modification of one from the past. It is a procedure based on principles of human behavior.

$$
=\frac{\sum_{i=1}^{n} f\left(N_{i}, S_{i}\right) \times w_{i}}{\sum_{i=1}^{n} w_{i}} \times 100(\%)
$$

where $\mathrm{N}$ is the new case, $\mathrm{S}$ is the case in the database, $\mathrm{n}$ is the number of variables for each case, $i$ is the variable from one to $n, f$ is the similarity function for a variable $i$ between cases $\mathrm{N}$ and $\mathrm{S}$, and $w_{i}$ are the weights of the variables (Kim et al. 2004).

Both of these methods are problematic because it is very difficult to describe the dependency of the variables mathematically. These methods offer a suitable solution if one already knows all the necessary mathematical functions.

\section{NN model}

NNs belong to the category of the biological (bioinspired) algorithms. The original aim of NN research was an attempt to understand and model the way humans think and the way in which the human brain works. Later, it was found that, for now, it is not possible to create the perfect model of the human brain. However, a NN is able to model some of its basic functions.

NNs allow an application where other models fail. It is about solving practical problems, which is a complicated deal for conventional computer models and in which there is no known algorithm. There must be available exemplifying data that adequately covers the problem area. These include pattern recognition, management area, prediction, analysis and transformation of data (Volná 2008).

\subsection{Biological and formal neurons}

Biological neuron is the fundamental building cell of the nervous system. Neurons serve for transmission, processing and storage of information necessary for the functioning of the organism. They consist of a body (soma) and the input and output transmission channels (dendrites and axons, respectively) (Volná 2008).

A formal neuron (Figure 1) is an essential element of the NN model.

Neuron Y has $n$ real inputs. Inputs are models of dendrites and they determine the input vector $\mathbf{x}=\left(\mathbf{x}_{\mathbf{1}}, \mathbf{x}_{\mathbf{2}}, \ldots\right.$ $\left.\mathbf{x}_{\mathbf{n}}\right)$. Inputs are evaluated by synaptic weights, which form the vector $\mathbf{w}=\left(\mathbf{w}_{\mathbf{1}}, \mathbf{w}_{\mathbf{2}}, \ldots . \mathbf{w}_{\mathbf{n}}\right)$, which may be negative also. The neuron has its own inner potential (Eq. 3), which is

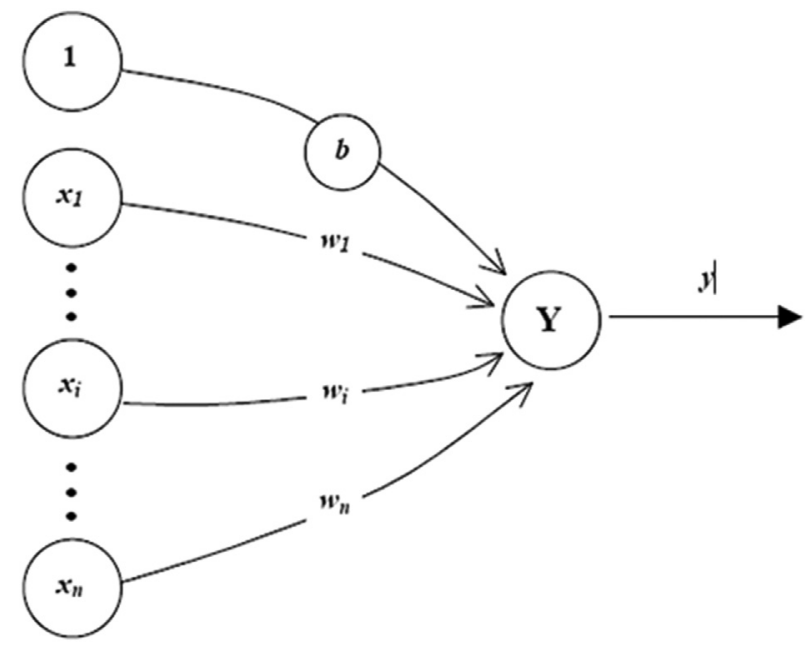

Fig. 1: Formal neuron (Volná 2008). 
computed as the weighted sum of the input values (Volná 2008).

$$
y \_ \text {in }=\sum_{i=1}^{n} w
$$

\subsection{NN model}

If we connect more neurons together, we will create a NN (Figure 2). Neurons in the network are combined into layers. The network consists of an input layer, which models the input data (parameters); the output layer, which models the results that are dependent on the inputs; and the inner layers that perform the calculation. The architecture of NN (the number of neurons and layers) determines the computing power and performance of the network.

\subsection{Backpropagation}

The backpropagation algorithm consists of three steps. Feed-forward step (input signal of training pattern is distributed from the input layer to the output layer), backpropagation step (error is distributed from the output layer to the input layer) and the step when the weights of the connections are updated. Weights for individual connections are at first randomly determined. During the feed-forward step, each neuron in the input layer receives the input signals (parameter values) and transfers the same to all the neurons of the inner layers using the selected transfer function. A more suitable transfer function in this case is the sigmoidal transfer function, which has a continuous first derivative at any point.

Each neuron in the inner layer calculates its activation and sends this signal to all the neurons in the output

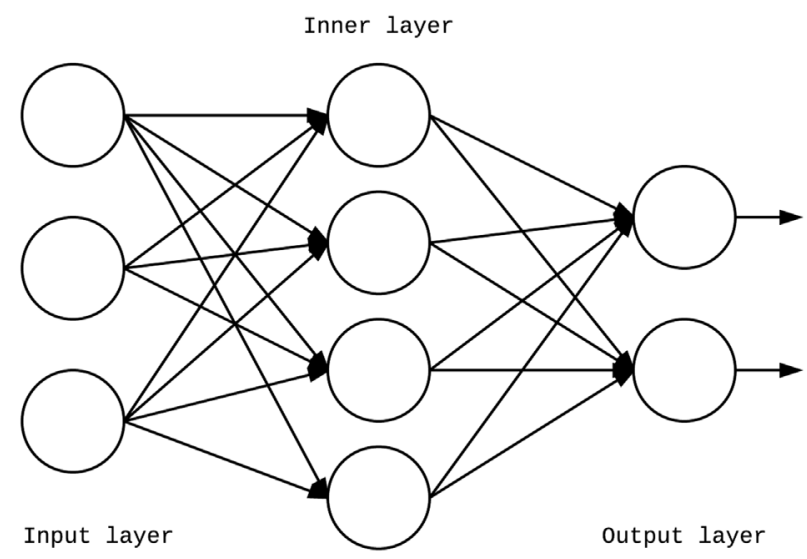

Fig. 2: Simple neural network. layer. Each neuron in the output layer calculates its activation, which corresponds to its actual output after the submission of the input pattern. Essentially, we get the feedback of our NN. This response shows us the error (deviation between the calculated values and the model), which is then allocated among the joints by adjusting their weights. For each adaptive pattern, we have to repeat this procedure. When the NN is properly trained, we can test our model by inputting testing patterns. If the NN is successfully tested, it can be used to estimate the output value based on our new input values.

\section{Example}

\subsection{Example explanation and first results}

For better understanding of the issue, especially the math behind it, an example has been created (Figures 3 and 4) through which the basic assumptions were verified. Given the complexity of operations associated with NNs, for future calculations, specialized software will be used; but, in this case, MS Excel was selected. Examples of steel structures are currently being processed and sufficient amount of data has not yet been recorded by field measurements. Therefore, the simple example of a worker transporting soil from one place to another was selected to demonstrate the basic assumptions.

Our assumption is that even a simple NN can easily simulate a simple process where we know all the variables. Finally, after training, we should finally get a model that would calculate the required value based on the insertion of known variables and which would be usable for any project.

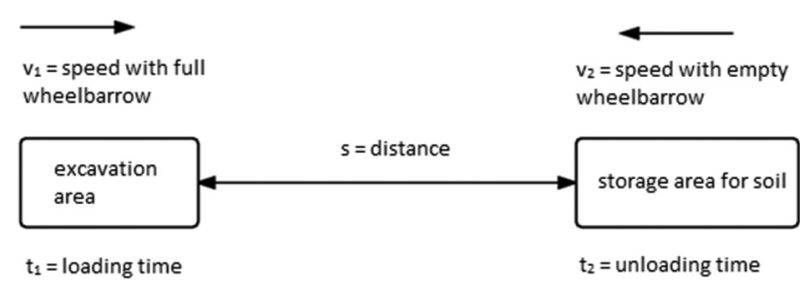

Fig. 3: Example - calculation of performance of a worker transporting soil.

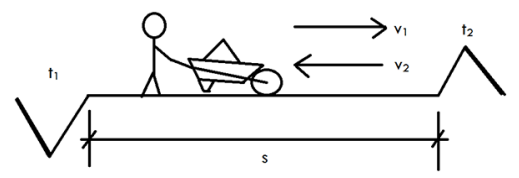

Fig. 4: Example - simple schema of a worker transporting soil. 
In this example (Figures 3 and 4), the dependent variable (performance) can be calculated using known formulas, and observation is not necessary. In order to calculate the performance of the worker, we need to know the loading time $\left(t_{1}\right)$, unloading time $\left(t_{2}\right)$, the distance $(s)$ and the speed with full $\left(v_{1}\right)$ and empty $\left(v_{2}\right)$ wheelbarrow.

Thus, 78 cases were created for learning and 50 cases for testing of the NN. In each case, the input variables were randomly generated, and the dependent variables were calculated by the algorithm (Figure 5).

The NN was created with five neurons as input layer (representing five inputs mentioned earlier) and one neuron as output layer (representing output - performance). The inner layer was chosen of the same size as

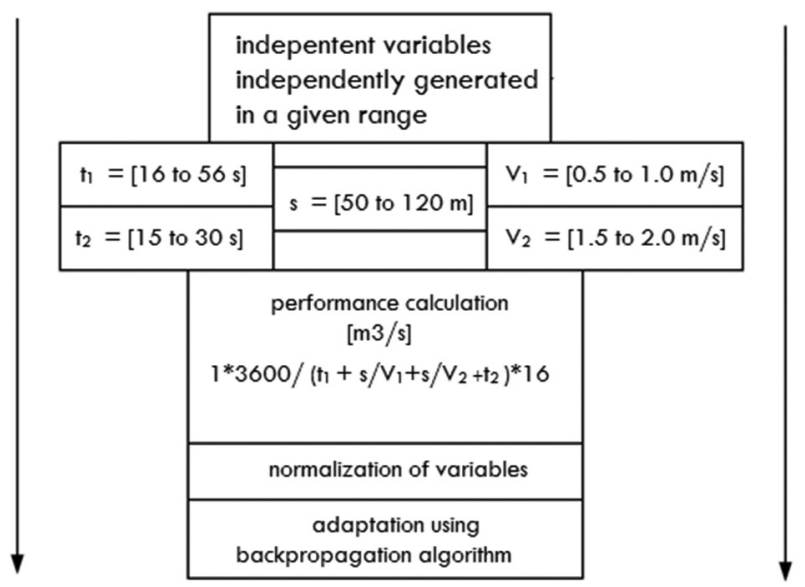

Fig. 5: Example algorithm. the input layer; therefore, it had five neurons. The input parameters and weights were randomly generated with continuous uniform distribution; in the future, these will be worked out with different distributions of probability.

The NN gradually processes individual cases. Every time a new input is recorded and evaluated, the value calculated by the NN and the value calculated by known procedures (according to the algorithm) are compared. The NN is considered sufficiently trained after its error is below the set limit.

Calculation performed in MS Excel demonstrated that the error decreases gradually but very slowly. For the network to be trained, it would require much more learning cycles to achieve accurate results. For the second set of calculations, we therefore used software specialized for training of NNs.

\subsection{Import to specialized software}

An example was imported into the software JustNN. Figure 6 shows the adaptation chart, which shows the number of cycles, and the gradually decreasing error. The error is the previously mentioned difference between the value calculated by the NN and by the known physical processes.

After the network adaptation using the software, a maximum error of $0.006519 \%$ was achieved. For the adaptation, 101 learning cycles of the earlier-mentioned 78 cases were needed. The calculation time was less than 1 minute.

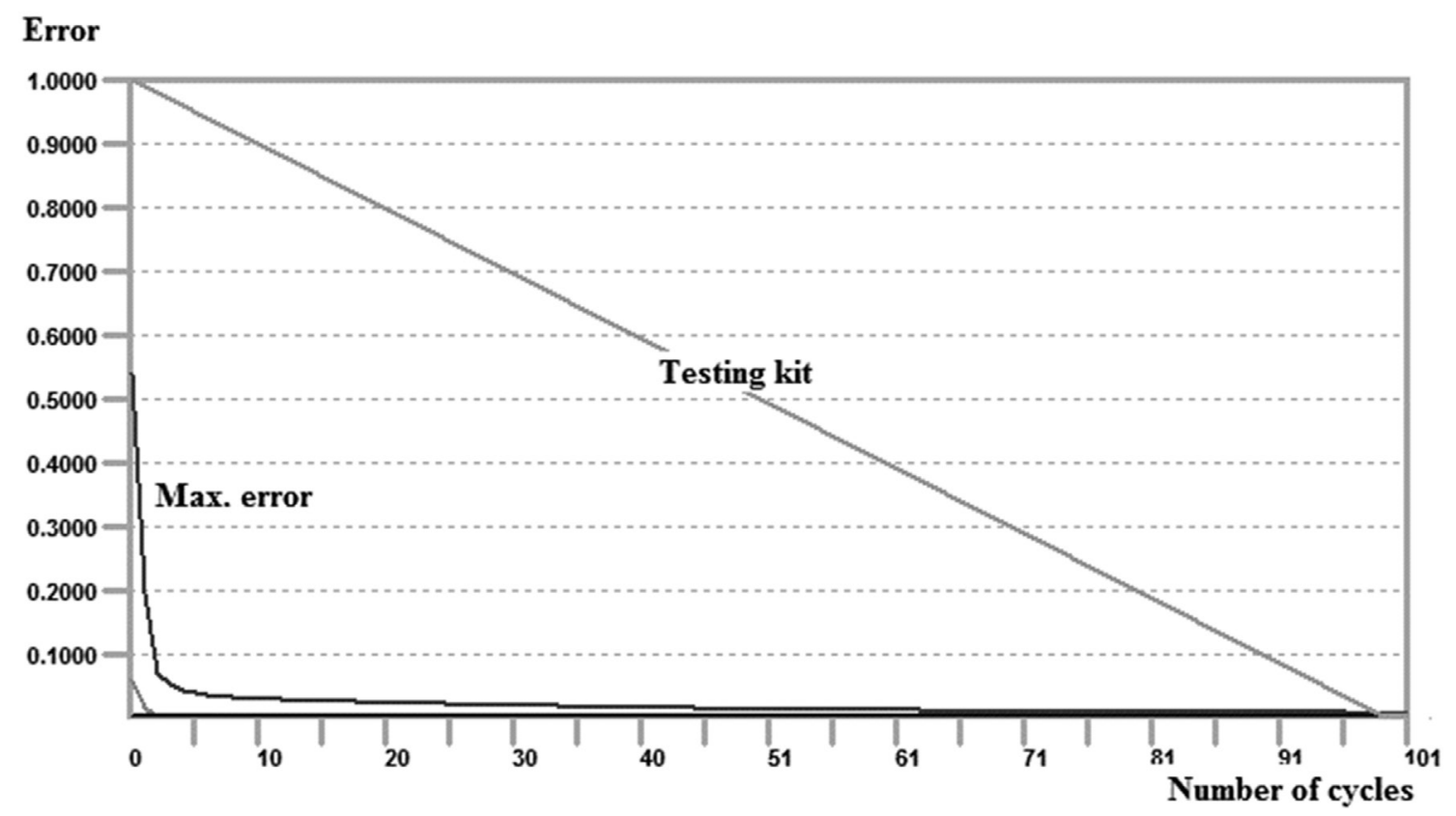

Fig. 6: Export of example adaptation chart of JustNN software. 


\section{Conclusions}

The following conclusions were reached:

- The higher the range of input values, the slower the network adapts but achieves more accurate results.

- The data can be cycled while adapting, but we need to enter enough data for this cycle to get as accurate a result as possible.

- After adapting the network, we can use the adapted weights to determine the degree of dependence of the dependent variable on the independent variables and to identify the independent variables that can be excluded from other calculations.

- Because of the high computational demands, special software must be used.

\section{Summary: direction of future research}

The first conclusions confirm the assumptions that even a simple NN can easily model a process for which we know all the variables. The problem, however, is that the learning of the network is very demanding for the large amount of data, which leads to the time-consuming nature of data acquisition. Therefore, the aim of further research should not be simply to compare the methods used to calculate the standard time with the method proposed by the authors using NNs. Instead, research should focus on reducing the time-consuming nature of data acquisition because this would exclude or restrict the biggest weakness of the methodology using NNs, i.e., its time-consuming feature. One option could be to partially replace the measured data with data that can be calculated.

\section{Acknowledgment}

This research was supported by a Student Grant Competition funding for project number SGS16/006/OHK1/1T/11.

\section{References}

Arditi, D., \& Tokdemir, O.B. (1999). Comparison of case-based reasoning and artificial neural networks. Journal of Computing in Civil Engineering, 13(3), pp. 162-169. doi: 10.1061/ (asce)0887-3801(1999)13:3(162).
Attalla, M., \& Hegazy, T. (2003). Predicting cost deviation in reconstruction projects: Artificial neural networks versus regression. Journal of Construction Engineering and Management, 129(4), pp. 405-411. doi: 10.1061/(asce)07339364(2003)129:4(405).

Berkhahn, V., \& Tilleke, S. (2008). Merging neural networks and topological models to re-engineer construction drawings. Advances in Engineering Software, 39(10), pp. 812-820.

Goh, B. (1998). Forecasting residential construction demand in Singapore: a comparative study of the accuracy of time series, regression and artificial neural network techniques. Engineering Construction and Architectural Management (Wiley-Blackwell), 5(3), pp. 261-275 [Business Source Complete, EBSCOhost, viewed 16 June, 2017].

Kim, G.-H., An, S.-H., \& Kang, K.-I. (2004). Comparison of construction cost estimating models based on regression analysis, neural networks, and case-based reasoning. Building and Environment, 39(10), pp. 1235-1242. doi: 10.1016/j.buildenv.2004.02.013.

Petroutsatou, K., Georgopoulos, E., Lambropoulos, S., \& Pantouvakis, J. P. (2012). Early cost estimating of road tunnel construction using neural networks. Journal of Construction Engineering and Management, 138(6), pp. 679-687. Available from: 10.1061/(ASCE)C0.19437862.0000479 on 16 June, 2017.

Volná, E. (2008). Neuronovésítě 1. Available at http://www1.osu. cz/ volna/Neuronove_site_skripta.pdf on 21 November, 2016.

Xu, L., Zhang, T., \& Ren, Q. (2015). Intelligent autofeedback and safety early-warning for underground cavern engineering during construction based on BP neural network and FEM. Mathematical Problems in Engineering [serial online], 2015(2015), pp. 1-8. Available at Academic Search Complete, Ipswich, MA. Accessed 16 June, 2017.

\section{Author biography}

Kateřina Petlíková is a student of doctoral program and a course instructor at the Department of Construction Technology, Faculty of Civil Engineering, at CTU in Prague, in the Czech Republic. She deals with the possibilities of using neural networks in the preconstruction phase. She is a researcher with several student grant projects.

Čeněk Jarský is an experienced professional in the field of software development for construction planning. For example, he developed the integrated project bidding, planning, management and quality control system named CONTEC. He is a university professor focused primarily on automatization in the construction field. An experienced researcher, he is the Head of Research Projects and Student Advisor. He is the author of monographs in the field of construction technology, automatization, preparation and management of construction, currently used at all faculties of construction engineering at Czech and Slovak technical universities and in practice. As a specialist, he has lent his expertise to important construction projects for Czech and foreign construction and investment companies. 